\title{
Reliability Theory Model and Expected Life Shortest Path in Stochastic and Time-Dependent Networks
}

\author{
Guo-zhen $\operatorname{Tan}^{1}$, Xiang-fu Xia ${ }^{1}$, and Wen $\mathrm{Gao}^{2}$ \\ 1 Department of Computer Science and Engineering, Dalian University of \\ Technology Dalian, Liaoning 116024, China ${ }^{\star \star \star}$ \\ gztan@dlut.edu.cn \\ 2 Institute of Computing Technology, Chinese Academy of Sciences, Beijing \\ 100080,China
}

\begin{abstract}
We consider the priori expected shortest path problem from a single origin to a single destination for each departure time in stochastic and time-dependent networks. Such problem requires more than standard shortest path techniques. First, we transform this problem into the problem of systemic reliability, and identify a weaker consistent reliability condition that insures the validity of generalized dynamic-programming method based on reliability priority. Furthermore, we propose a new dominance discriminance, which makes the parameter in traditional discriminance drop from two dimensions to one dimension and reduces the possibility of not doing dominance compare among paths. Finally, we present the expected life shortest path algorithm.
\end{abstract}

\section{Introduction}

The study of routing problem in stochastic time-dependent networks is of timely importance, as stochasticity in traffic networks is both conceptually recognized and experimentally proved [1]. A stochastic time-dependent network is a network where link travel times are random variables with time-dependent distributions [2].

Many researchers have tried to use different ways to solve the stochastic shortest path problem, including reducing the search space to find feasible solutions. A method proposed by Alexopoulos is based on iterative partitioning of the network state space and provides bounds that improve each iteration and eventually become equal to the respective measures related to the shortest paths in networks 3 . We have studied the shortest path algorithm in time-dependent networks, and got some valuable conclusions [4]. Hall has studied a specific variant of optimal routing problem in stochastic and time-dependent networks where a routing policy depends only on arrival times at decision nodes [5]. Wellman, Ford, and Larson have demonstrated that standard search algorithms could find

$\star \star \star$ This work was supported in part by Grand 99025 of Ministry of Education and Grand 9810200104 of Liaoning Science Foundation, China. 
the shortest path when arc travel times have weaker stochastic consistency [6]. This property in turn justifies a generic pruning method using stochastic dominance. But the dominance discriminance used in 6] contains two parameters. J.Boyan and M.Mitzenmacher [7] have studied the problem of the scheduling policy in stochastic networks, using the reliability theory. They consider the waiting time for buses, and they present the optimal scheduling algorithm when the waiting times are independent random variables with increasing failure rate. But they cannot realize this algorithm.

In this paper, we study the shortest path problem in stochastic and timedependent networks, an important problem in communication, transportation and internet systems. Using reliability theory, this problem is transformed into finding a path based on reliability priority. We consider the travel time of the entire path from source node to destination node, and our goal is to select a statistically optimal path. We identify a weaker consistent reliability condition that insures the validity of generalized dynamic-programming method based on reliability theory. The consistent reliability property essentially is to make use of reliability theory to describe the First-in-First-out property of every arc in the networks. Furthermore, a new discriminance is proposed to reduce the two parameters in extant discriminance 8] to one, which makes this discriminance feasible and reduces the possibility of not doing compare among paths. Finally, we present the Expected-Life-Shortest-Path algorithm, which can be used to solve problems of considerably great size.

\section{Preliminary Knowledge}

We first briefly introduce the basic concepts associated with reliability theory, which makes this paper easier to understand. Further information can be found in texts such as [8] or [9].

We use a non-negative random variable $X$ to denote a system's life, where $X$ has cumulative distribution function $F(t)$. Then we define the survival function of this system to be $S(t)=1-F(t)$. Obviously, $S(t)$ describes the system's viability in the time interval between 0 and $t$.

The system's mean life is

$$
E X=\int_{0}^{\infty} t d F(t)=\int_{0}^{\infty} d F(t) \int_{0}^{t} d x=\int_{0}^{\infty} d x \int_{x}^{\infty} d F(t)=\int_{0}^{\infty} S(t) d t
$$

The mean residual life of the system at time $t$ is $E[X-t \mid X \geq t]$, which is dependent of $t$.

Let $d=\sup \{t \mid S(t)>0\}$, and $D=[0, d)$.

Suppose $f(t)$ is the probability density function of $X$, then we define the failure rate of this system to be

$$
r(t)=\frac{f(t)}{S(t)}, \quad t \in D
$$

On second thoughts, we can get (3) and (4) 


$$
\begin{aligned}
& r(t)=-\frac{d}{d t} \ln S(t) \\
& S(t)=e^{-\int_{0}^{t} r(x) d x}
\end{aligned}
$$

\section{Reliability Theory Model}

Since the reliability theory is the theory focusing on a system's stochastic life, we first depict the stochastic and time-dependent network as a system, called STDN$R$ network system. Then we transform the shortest path problem in stochastic and time-dependent networks into the problem of the system's life. We construct a system consisting of the entire public transit network together with travel time $T$, where $T$ is the time that a traveller takes from the source node to the destination node, thus $T$ is the system's life. To give expression to the dynamic process of the system life, we introduce the concept of path life. The life of a path is the travel time that a traveller has spent from the source node to node $v_{i}$.

In the $S T D N-R$ network system, the system's life is stochastic due to the fact that the arcs' lives (i.e.costs) are stochastic. We use a non-negative random variable $X$ to denote the system's life. The optimal goal is to manage to make the system's life as short as possible. That is to say, we expect to find a $t$ small enough to make the system's viability lowest (that is failure rate maximal) in time range from 0 to $t$.

In this system, the traveller's arriving at the last node of the current path leads to an event called failure. The initial failure time of a system is the time when the traveller starts at the source node of the network system; the expected failure time of a system is the expected time when the traveller reaches the destination node of the network system; the initial failure time of an arc is the time when the traveller leaves the tail node of the arc; the expected failure time of an arc is the expected time when the traveller arrives at the head node of the arc.

\subsection{STDN-R Network System}

In this paper, the reliability theory model in stochastic and time-dependent networks is called $S T D N-R$ network system. Its formalization expression is $S T D N-R=(V, A, L, \Phi)$, where $V$ is a non-empty set of nodes; $A \subseteq V \times V$ is a finite set of $\operatorname{arcs} ; L$ is a finite set of the possibly least life of every arc; $\Phi=\left\{\varphi_{m}^{I}(t) \mid I=\left(t_{i j}^{m-1}, t_{i j}^{m}\right],\left\langle v_{i}, v_{j}\right\rangle \in A, 1 \leq m \leq N_{i j}\right\}$. Thereinto, $m$ is a number assigned to certain stream,the numbers are assigned based on their departure schedule at $v_{i}$ along the $\operatorname{arc}\left\langle v_{i}, v_{j}\right\rangle$, and the quantity of all the streams on the $\operatorname{arc}\left\langle v_{i}, v_{j}\right\rangle$ is called traffic $N_{i j}, m \in\left\{1,2, \ldots, N_{i j}\right\} ; I=\left(t_{i j}^{m-1}, t_{i j}^{m}\right]$ is the time range that a stream arrives at $v_{i}$, where this stream is certain stream on the former arc adjacent to node $v_{i} ; t_{i j}^{m}$ is the departure time of $m$ at $v_{i} ; \varphi_{m}^{I}(t)$ is 
the density function of the probability distribution which $t$ subjects to, where $t$ is the time arriving at $v_{j}$ for $m$ along the $\operatorname{arc}\left\langle v_{i}, v_{j}\right\rangle$.

This is a abstract model of the network-optimizing problem, which has different meanings in different fields. For example, in bus networks, a stream represents a bus on certain arc; in computer networks, a stream represents a data package on certain link.

Let the sequence $\left\{s=v_{0}, v_{1}, v_{2}, \ldots, v_{h-1}, v_{h}=d\right\}$ represent the path from $s$ to $d$ and denote it as $P_{s d}$, where $\left\langle v_{j-1}, v_{j}\right\rangle \in A \quad 1 \leq j \leq h$. Let the expected failure time of path $P_{s d}$ be $E T\left(P_{s d}\right)$, and let its expected life be be $E L\left(P_{s d}\right)$. The relationship between them is

$$
E T\left(P_{s d}\right)=E L\left(P_{s d}\right)+\mu_{0}
$$

where $\mu_{0}$ is the initial failure time.

In this network system, the problem of expected life shortest path is a priori expected shortest path problem from a single origin to a single destination for each departure time.

\subsection{Consistent Reliability Property}

Definition 1. In $S T D N-R$ network system, let $l_{i j}(x)$ represent the actual life of the $\operatorname{arc}\left\langle v_{i}, v_{j}\right\rangle$ whose initial failure time is $x$. Let $0 \leq s \leq t$, for $\forall\left\langle v_{i}, v_{j}\right\rangle \in A$, if the network satisfies

$$
r\left(s+l_{i j}(s)\right) \geq r\left(t+l_{i j}(t)\right)
$$

we say that the network has the consistent reliability property.

This definition makes it clear that even though the initial failure time is late, failure rate will not increase. That is, the consistent reliability property essentially is to make use of reliability theory to describe the First-in-First-out property of every arc in the network.

\subsection{Reliability Priority}

Reliability Priority. The reliability priority describes the properties of certain path in the network, while the consistent reliability property describes the characteristics of each arc in the entire network.

Definition 2. Let $r_{1}(z)$ represent the failure rate of system at time $z$ along the path $P_{1}$, and let $r_{2}(z)$ represent the failure rate of system at time $z$ along the path $P_{2}$. For $\forall z$, if $r_{1}(z) \geq r_{2}(z)$, we say that path $P_{1}$ has the reliability priority relative to path $P_{2}$, i.e. $P_{1} \leq_{a d} P_{2}$.

From (3) and (4), we can know that $r_{1}(z) \geq r_{2}(z) \Longleftrightarrow S_{1}(z) \leq S_{2}(z)$, so we can get the property as follows.

Deduction 1. Let $S_{1}(z)$ represent the survival function of system at time $z$ along the path $P_{1}$, and let $S_{2}(z)$ represent the survival function of system at time $z$ along the path $P_{2}$. Then we can get, for $\forall z, S_{1}(z) \leq S_{2}(z) \Longleftrightarrow P_{1} \leq a d P_{2}$. 
In fact, the intuitionistic meaning of reliability priority is to say, if the expected life of path $P_{1}$ is not longer than that of path $P_{2}$, then path $P_{1}$ has the reliability priority relative to path $P_{2}$.

Theorem 1. $P_{1} \leq_{a d} P_{2} \Longleftrightarrow E L\left(P_{1}\right) \leq E L\left(P_{2}\right) \Longleftrightarrow E T\left(P_{1}\right) \leq E T\left(P_{2}\right)$.

Proof: We first prove $P_{1} \leq_{a d} P_{2} \Longleftrightarrow E L\left(P_{1}\right) \leq E L\left(P_{2}\right)$.

$\Longrightarrow$ : Given that $P_{1} \leq_{a d} P_{2}$, from Deduction 1 , for $\forall z$, we have $S_{1}(z) \leq S_{2}(z)$. Then, from (11), we can conclude that $E L\left(P_{1}\right)=\int_{0}^{\infty} S_{1}(z) d z \leq \int_{0}^{\infty} S_{2}(z) d z=$ $E L\left(P_{2}\right)$, i.e. $E L\left(P_{1}\right) \leq E L\left(P_{2}\right)$.

$\Longleftarrow$ : Obviously.

So $P_{1} \leq_{a d} P_{2} \Longleftrightarrow E L\left(P_{1}\right) \leq E L\left(P_{2}\right)$.

From (5), we can conclude that $E L\left(P_{1}\right) \leq E L\left(P_{2}\right) \Longleftrightarrow E T\left(P_{1}\right) \leq E T\left(P_{2}\right)$. Proof is end.

Dominance Discriminance based on Reliability Theory. For the failure time of an arc subjects to exponential distribution $\operatorname{EXP}(M, \lambda)$, where $M$ is the possibly earliest failure time of the arc and $\lambda$ is the mean residual life of the arc overstep $M$, the traditional dominance discriminance contains two parameters [8], i.e. $M, \lambda$ : $A$ has stochastic dominance relative to $B$, iff $M_{A} \leq M_{B}$ and $\lambda_{A} \leq \lambda_{B}$. But when $M_{A} \leq M_{B}$ and $\lambda_{A} \geq \lambda_{B}$, or $M_{A} \geq M_{B}$ and $\lambda_{A} \leq \lambda_{B}$, $A$ can't do compare with $B$. In this paper, a new discriminance is proposed to reduce the two parameters in extant discriminance [8] to one, which reduces the possibility of not doing compare among paths.

Now, we consider the discriminance based on reliability theory when the probability distribution is exponential distribution $\operatorname{EXP}(M, \lambda)$. Because the distribution function is $F(t)=1-e^{-\frac{t-M}{\lambda}} \quad t \geq M$, the failure rate is $r(t)=\frac{1}{\lambda}$. Let the two paths that can reach the destination node be $P$ and $P^{\prime}$ respectively. The failure time on the last arc of path $P$ that satisfies $\operatorname{EXP}\left(M_{1}, \lambda_{1}\right)$ has probability $p_{1}$. The one that satisfies $\operatorname{EXP}\left(M_{2}, \lambda_{2}\right)$ has probability $p_{2}$ and so on. Reorder the exponential distribution sequence of $P$ and let any adjacent terms $\operatorname{EXP}\left(M_{i}, \lambda_{i}\right)$ and $\operatorname{EXP}\left(M_{i+1}, \lambda_{i+1}\right)$ satisfy $\lambda_{i} \leq \lambda_{i+1}$. Do the same to path $P^{\prime}$ to obtain

$P:\left(\operatorname{EXP}\left(M_{1}, \lambda_{1}\right), p_{1}\right),\left(\operatorname{EXP}\left(M_{2}, \lambda_{2}\right), p_{2}\right), \cdots,\left(\operatorname{EXP}\left(M_{i}, \lambda_{i}\right), p_{i}\right), \cdots$

$P^{\prime}:\left(E X P\left(M_{1}^{\prime}, \lambda_{1}^{\prime}\right), p_{1}^{\prime}\right),\left(E X P\left(M_{2}^{\prime}, \lambda_{2}^{\prime}\right), p_{2}^{\prime}\right), \cdots,\left(E X P\left(M_{i}^{\prime}, \lambda_{i}^{\prime}\right), p_{i}^{\prime}\right), \cdots$

Using the notation $e p_{i}=\sum_{k=1}^{i} p_{k}$ and the above two sequences, we can get the sequences of partial sums of probability as follows:

$P: e p_{1}, e p_{2}, \cdots, e p_{i}, \cdots, 1$

$P^{\prime}: e p_{1}^{\prime}, e p_{2}^{\prime}, \cdots, e p_{i}^{\prime}, \cdots, 1$

Let us rearrange the elements of the sequences of partial sums of probability in ascending order. We obtain the following sequence:

$m p_{1}, m p_{2}, \cdots, m p_{i}, \cdots, 1$.

Taking any element $m p_{i}$ from above sequence, and letting $q=\min _{k}\{k \mid$ $\left.e p_{k} \geq m p_{i}\right\}, q^{\prime}=\min _{k}\left\{k \mid e p_{k}^{\prime} \geq m p_{i}\right\}$.

For $\forall m p_{i}$, if $\lambda_{q} \leq \lambda_{q^{\prime}}^{\prime}$, then $P \leq_{a d} P^{\prime}$.

Theorem 2. The dominance discriminance based on reliability theory is true. 
Proof: For each $\left[m p_{i-1}, m p_{i}\right]\left(i \geq 1, m p_{0}=0\right)$ in the probability range $[0,1]$, there exist $\lambda_{q} \leq \lambda_{q^{\prime}}^{\prime}$, so for $\forall z$, we can get $r(z)=\frac{1}{\lambda_{q}} \geq \frac{1}{\lambda_{q^{\prime}}^{\prime}}=r^{\prime}(z)$ in $\left[m p_{i-1}, m p_{i}\right]$, i.e. $P \leq_{a d} P^{\prime}$ in $\left[m p_{i-1}, m p_{i}\right]$. Then let different probability interval $\left[m p_{i-1}, m p_{i}\right]$ multiply their singly relevant failure rate, and then find the sum. We conclude that $r(z)=\Sigma_{i}\left(m p_{i}-m p_{i-1}\right) \cdot \frac{1}{\lambda_{q}} \geq \Sigma_{i}\left(m p_{i}-m p_{i-1}\right) \cdot \frac{1}{\lambda_{q^{\prime}}^{\prime}}=r^{\prime}(z)$. In fact, $r(z)$ represents the failure rate of system at time $z$ along path $P, r^{\prime}(z)$ represents the failure rate of system at time $z$ along path $P^{\prime}$. So, for $\forall z, r(z) \geq r^{\prime}(z)$, from Definition 2 we can conclude $P \leq_{a d} P^{\prime}$. Proof is end.

\subsection{The Monotonicity Principle}

Theorem 3. In the given $S T D N-R$ network system, let $P_{1}=\left\{s=v_{0}, v_{1}, v_{2}, \cdots, v_{i-1}, v_{i}\right\}, P_{1}^{\prime}=\left\{s=v_{0}, v_{1}, v_{2}, \cdots, v_{i-1}, v_{i}, v_{j}\right\}, P_{2}=$ $\left\{s=v_{0}, v_{1}^{\prime}, v_{2}^{\prime}, \cdots, v_{i-1}^{\prime}, v_{i}\right\}, P_{2}^{\prime}=\left\{s=v_{0}, v_{1}^{\prime}, v_{2}^{\prime}, \cdots, v_{i-1}^{\prime}, v_{i}, v_{j}\right\}$. If the STDN$R$ network has the consistent reliability property, then $P_{1} \leq{ }_{a d} P_{2} \Longrightarrow P_{1}^{\prime} \leq_{a d} P_{2}^{\prime}$.

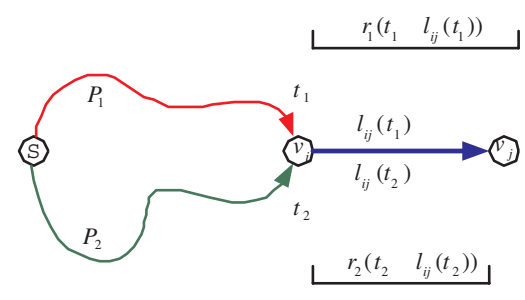

Fig. 1. Monotonicity Demonstration

Proof: Let $t_{1}$ be the failure time of path $P_{1}$ and $t_{2}$ be the failure time of path $P_{2}$. Because of $P_{1} \leq{ }_{a d} P_{2}$, we can conclude there must be $t_{1} \leq t_{2}$ according to Theorem 1. Let $r_{1}(z)$ represent the failure rate of system at time $z$ along path $P_{1}$, $r_{2}(z)$ represent the failure rate of system at time $z$ along path $P_{2}$, and let $l_{i j}(x)$ represent the actual life of the arc $\left\langle v_{i}, v_{j}\right\rangle$ whose initial failure time is $x$. Because $S T D N-R$ network has the consistent reliability property, from Definition 1 , we can conclude $r_{1}\left(t_{1}+l_{i j}\left(t_{1}\right)\right) \geq r_{2}\left(t_{2}+l_{i j}\left(t_{2}\right)\right)$. Because the above conclusion is always tenable for starting from source node at every time, we must have $P_{1}^{\prime} \leq_{a d} P_{2}^{\prime}$

\section{Calculation of Expected Failure Time and Expected Life}

Let event $A_{i j}^{m}$ represent a trip in which a traveller takes bus $m$ at node $v_{i}$ along arc $\left\langle v_{i}, v_{j}\right\rangle, m \in\left\{1,2, \cdots, N_{i j}\right\}$, where $m$ is a number assigned to the 
bus starting from $v_{i}$ to $v_{j}$. The numbers are assigned based on their departure schedule. $N_{i j}$ represents the total number of buses travelling on $\operatorname{arc}\left\langle v_{i}, v_{j}\right\rangle$. Then the $\operatorname{set}\left\{A_{i j}^{m} \mid 1 \leq m \leq N_{i j}\right\}$ is the partition of the sample space $\Omega$. That is,

$$
A_{i j}^{m} \cap A_{i j}^{n}=\Phi, \bigcup_{m=1}^{N_{i j}} A_{i j}^{m}=\Omega \text { and } \operatorname{Pr}\left(A_{i j}^{m}\right) \geq 0 \text { where } 1 \leq n \neq m \leq N_{i j} .
$$

If the first bus that departs from node $v_{i}$ at time $\mu_{i}$ is $m$, then $\operatorname{Pr}\left(A_{i j}^{m}\right)=$ $1, \operatorname{Pr}\left(A_{i j}^{n}\right)=0$, where $1 \leq n \neq m \leq N_{i j}, \mu_{i}$ is the initial failure time of arc $\left\langle v_{i}, v_{j}\right\rangle$.

Assuming that we have already known $\operatorname{Pr}\left(A_{i j}^{n}\right)$, where $1 \leq n \leq N_{i j}$, the time that bus $n$ arrives at $v_{j}$ satisfies $t \sim \varphi_{n}(t)$, where $t_{i j}^{n-1}<\mu_{i} \leq t_{i j}^{n}$. The arrival time of bus $m$ which departs from $v_{j}$ and arrives at $v_{k}$ satisfies $t \sim \varphi_{m}(t)$, where $t_{j k}^{m-1}<\mu_{j} \leq t_{j k}^{m}$, then $\operatorname{Pr}\left(A_{j k}^{m} \mid A_{i j}^{n}\right)=\int_{t_{j k}^{m-1}}^{t_{j k}^{m}} \varphi_{n}(t) d t$. According to the total probability formula, we can get

$$
\operatorname{Pr}\left(A_{j k}^{m}\right)=\sum_{n=1}^{N_{i j}} \operatorname{Pr}\left(A_{i j}^{n}\right) \cdot \operatorname{Pr}\left(A_{j k}^{m} \mid A_{i j}^{n}\right)=\sum_{n=1}^{N_{i j}} \operatorname{Pr}\left(A_{i j}^{n}\right) \cdot \int_{t_{j k}^{m-1}}^{t_{j k}^{m}} \varphi_{n}(t) d t .
$$

Now that, we have known path $P=\left\{v_{0}, v_{1}, \cdots, v_{i-1}, v_{i}, v_{i+1}, \cdots, v_{h-1}, v_{h}\right\}$ and its initial failure time $\mu_{0}$, then the calculation method of the expected failure time $E T(P)$ and the expected life $E L(P)$ as follows:

$$
\begin{cases}\operatorname{Pr}\left(A_{01}^{m}\right)=1, & \begin{array}{l}
\text { if } t_{01}^{m-1}<\mu_{0} \leq t_{01}^{m} \\
\operatorname{Pr}\left(A_{01}^{m}\right)=0,
\end{array} \\
\cdots \quad \cdots \quad \cdots \quad \cdots & \text { else } \\
\operatorname{Pr}\left(A_{i(i+1)}^{m}\right)=\sum_{n=1}^{N_{(i-1) i}} \operatorname{Pr}\left(A_{(i-1) i}^{n}\right) \cdot \int_{t_{i(i+1)}^{m-1}}^{t_{i(i+1)}^{m}} \varphi_{n}(t) d t & \\
\cdots \quad \cdots \quad \cdots \quad \cdots & \\
E T\left(P_{1 h}\right)=\sum_{m=1}^{N_{(h-1) h}} \operatorname{Pr}\left(A_{(h-1) h}^{m}\right) \cdot \int t \cdot \varphi_{m}(t) d t & \\
E L\left(P_{1 h}\right)=E T\left(P_{1 h}\right)-\mu_{0} & \end{cases}
$$

\section{$5 \quad$ RELSP Algorithm}

If one path reached node $v_{i}$ has the reliability priority relative to another, then the consistent reliability property insures that the latter cannot become one part of the optimal path. According to the dominance discriminance based on reliability theory, RELSP algorithm deletes the sub-paths without reliability priority. If there are lots of paths without reliability priority, then the algorithm will not use bigger storage space and computing time.

Input: an expanded stochastic and time-dependent network, which has consistent reliability property; source node $s$; destination node $d$; initial failure time $\mu_{0}$; a non-increasing utility function $u(x)$.

Output: the optimal path from source node $s$ to destination node $d$. 


\section{Data structures:}

open: the set of expanded nodes. Let each expanded node only map to one path. These expanded nodes are represented by the numbers assigned to each node. Each node can map to multiple expanded nodes. Sort the elements in open according to utility function $u(x)$.

$S E T(v)=\{w \mid\langle v, w\rangle \in A\}$ represents the set of all nodes adjacent to node $v$.

$D P_{v}$ saves all paths with reliability priority from source node $s$ to node $v$, where $v \in V$.

\section{Algorithm:}

Step1: Initializes the expanded node that source node $s$ is corresponding to, and put the expanded node into open;

Step2: If open is non-empty, goto Step3; otherwise, goto Step9;

Step3: Take out the element $q$ whose value of the utility function is maximal from open, and let the last node that $q$ is corresponding to be $v$, if $v$ is destination node $d$, goto Step9; otherwise, goto Step4;

Step4: Review all the arc $\langle v, w\rangle$ that is derivate from node $v$, and get the set $\operatorname{SET}(v)$ of nodes adjacent to $v$;

Step5: If $\operatorname{SET}(v)$ is non-empty, then take out a node $w$ from $S E T(v)$, and expand the path corresponding to $q$ to $w$, and get a new expanded node $n u m$, goto step6; otherwise, goto step2;

Step6: If there is a path has the reliability priority relative to the path that num is corresponding to, then the algorithm do nothing, goto Step5; otherwise, put the path that num is corresponding to into $D P_{w}$, goto Step7;

Step7: Calculate the expected failure time and expected life that num is corresponding to;

Step8: According to utility function $u$ insert num into the appropriate place in open; goto step5;

Step9: Output the optimal path from source node $s$ to destination node $d$, the algorithm is end.

Theorem 4. If the input network of the RELSP algorithm has consistent reliability property, and the utility function $u(x)$ is non-increasing, then the $R E L S P$ algorithm will find the optimal path from source node $s$ to destination node $d$.

Proof: During the iteration, the algorithm divided path spaces having been searched into two parts: one part is the space of deleted paths, and let the set of nodes that the space contains be $N D P$; the other part is the space of saved paths, and let the set of nodes that the space contains be DP. But, there still exists path space having not been searched when the algorithm is end, and let the set of nodes that the space contains be NSP. Thus, $V$ is divided into three subclasses, the relationship among them is shown by Fig. 2. Obviously, $\{s, d\} \in D P \cap N D P \cap N S P$.

The terminative condition of the algorithm is that open is empty or searching $d$. The first condition means that there are no path from $s$ to $d$ at all; We merely prove that the second condition means that we have found the optimal path from $s$ to $d$. 


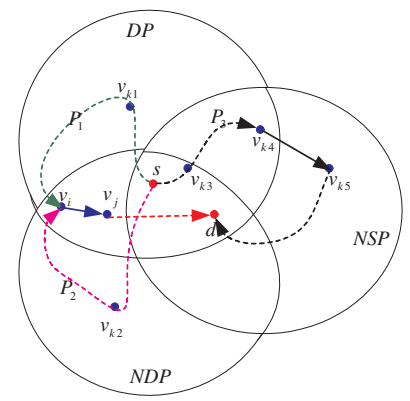

Fig. 2. The Validity of RELSP algorithm

Let $P_{1}$ be the sub-path passing through $v_{k 1} \in D P-N D P$ and reaching $v_{i} \in D P \cap N D P, P_{2}$ is the sub-path passing through $v_{k 2} \in N D P-D P$ and reaching $v_{i} \in D P \cap N D P$, and $P_{1} \leq_{a d} P_{2}$. During the iteration, the algorithm deleted sub-path $P_{2}$. Then we prove the validity of the deleting operation as follows. Let the node set adjacent to $v_{i}$ be $S E T\left(v_{i}\right)=\left\{v_{j} \mid\left\langle v_{i}, v_{j}\right\rangle \in A\right\}$. For $\forall v_{j} \in S E T\left(v_{i}\right)$, expand $P_{1}$ and $P_{2}$ respectively, thus we can get $P_{1}^{\prime}$ and $P_{2}^{\prime}$. Because $P_{1} \leq_{a d} P_{2}$, we can conclude $P_{1}^{\prime} \leq_{a d} P_{2}^{\prime}$ according to Theorem 3 . Therefore, $P_{2}$ will never become the sub-path of the optimal path from $s$ to $d$, so we may delete it.

In addition, the paths not being searched when the algorithm is end will not become the optimal path. This because that if we want to search these paths, we can carry our point only by expanding the sub-paths that are corresponding to those residual elements in open. For example, as Fig. 2 is shown, let $P_{3}^{\prime}=$ $\left\{s, \cdots, v_{k 3}, \cdots, v_{k 4}, v_{k 5}, \cdots, d\right\}$ be certain path having still not been searched when algorithm is end, if we want to search $P_{3}^{\prime}$, then we must expand $P_{3}=$ $\left\{s, \cdots, v_{k 3}, v_{k 4}\right\}$ to $v_{k 5}$, where $P_{3}$ is the path corresponding to certain element num in open, $\cdots$, continue to expand till $d$. However, in open, num is behind the element corresponding to the path just searched, i.e.the value of utility function of $P_{3}$ is not bigger than that of the path just searched. We get $P_{3}^{\prime}$ by expanding $P_{3}$, so the value of utility function of $P_{3}^{\prime}$ is smaller than that of $P_{3}$. Thereby, the value of utility function of $P_{3}^{\prime}$ must not be bigger than that of the path just searched, i.e. it is impossible for $P_{3}^{\prime}$ to become the optimal path. As well as, during the course of iterative, the algorithm takes out the optimal element from open every time. Therefore, the path that has just been found must be the optimal path from $s$ to $d$. Proof is end.

Finally, we show an application example of the RELSP algorithm in [10], and make an comparison with the PFS-Dominance algorithm of Wellman (see [6]), and our RELSP algorithm is proved very efficient. 


\section{Conclusions}

Compared with traditional deterministic networks, stochastic and timedependent networks have more applications, in particular, in the fields such as wireless communications, intelligent transportation systems, and the Internet. All these new network applications have stochastic and time-dependent properties. Therefore, research on these stochastic and time-dependent networks is very important. Besides transportation networks, our study results should be applicable to other similar networks, such as computer networks and communication networks. Note that how to determine reliability priority is not an essential and necessary condition but a necessary condition. If an essential and necessary condition can be found, the search space can be further reduced.

\section{References}

1. J.A. Bottom, Anticipatory Consistent Route Guidance, Ph.D.thesis, Massachusetts Institute of Technology, Step, 2000.

2. Song Gao; Chabini, I. Optimal routing policy problems in stochastic timedependent networks part I: framework and taxonomy. Intelligent Transportation Systems, 2002. Proceedings. The IEEE 5th International Conference on, 2002.Page(s): 549-554.

3. Christos Alexopoulos, "State Space Partitioning Methods for Stochastic Shortest Path Problems," Networks, vol. 30, no. 1, 1997, pp. 9-21.

4. Tan Guozhen, Gao Wen. Shortest path algorithm in time-dependent networks. Chinese J.Computers, 2002, 25(2) 165-172 (in Chinese).

5. R.W. Hall, "The fastest path through a network with random time-dependent travel times", Transportation Science, vol.20, no.3, pp.91-101, 1986.

6. Michael P. Wellman, Matthew Ford, and Kenneth Larson, "Path Planning under Time-Dependent Uncertainty," Proc. Eleventh Conf. On Uncertainty in Artificial Intelligence, Aug. 1995, pp. 532-539.

7. Justin Boyan and Michael Mitzenmacher.Improved Results for Route Planning in Stochastic Transportation Network. SODA. 2001.

8. D.Stoyan. Comparison Methods for Queues and Other Stochastic Models. John Wiley and Sons, New York, 1983.

9. S.Ross. Stochastic Process. John Wiley and Sons, New York, 1983.

10. TAN Guo-zhen and XIA Xiang-fu. Algorithms for routing in stochastic and timedependent networks based on reliability theory. Research report of Dept. of Computer Science and Engineering, Dalian University of Technology. No 200201. 